\title{
Optimal insurance coverage of low-probability catastrophic risks
}

\author{
Alexis Louaas* and Pierre Picard ${ }^{\dagger}$
}

February 12, 2020

\begin{abstract}
Catastrophic risks are often characterised by a low probability, a high severity and a large number of affected individuals. Taking these specificities into account, we analyse the capacity of insurance contracts to provide coverage for those risks, independently from the market failures frequently observed in practice. On the demand side, we characterise individual preferences under which the willingness to pay for the coverage of large losses remains significant, although their occurrence probability is very small. On the supply side, the correlation between individual losses affects the insurance pricing through the insurers' cost of capital. Analysing the interaction between demand and supply yields the key determinants of insurability and of a socially optimal risk sharing strategy.
\end{abstract}

Keywords: Disaster insurance, catastrophic risk, risk aversion, capital costs.

JEL classification: D81, D86, G22, G28.

${ }^{*}$ CREST-Ecole Polytechnique, France. E-mail : alexis.louaas@polytechnique.edu

${ }^{\dagger}$ CREST-Ecole Polytechnique, France. E-mail : pierre.picard@polytechnique.edu.

Address for correspondence: Ecole Polytechnique, Department of Economics, 91128

Palaiseau Cedex, France 


\section{Introduction}

The insurability of catastrophic risks is at the heart of many economic policy debates and the aggregate consequences of these risks, be they natural or man-made, are often emphasised. Rietz (1988) and Barro (2006) for example, documented significant effects on asset prices while Gourio (2012) and Farhi \& Gabaix (2015) highlighted effects on business cycles and interest rates. In the context of global climate change, Weitzman (2009) also illustrated the potentially disastrous consequences of uninsured catastrophic risks on welfare. The coverage of these risks is therefore a crucial question, at the intersection of government decision-making and market mechanisms.

We are particularly concerned with very low probability accidents, typically not insured by private markets because of the large exposure they induce for insurance providers. For instance, large-scale nuclear disasters, such as Fukushima-Daiichi, are estimated to occur with an annual probability of the order of $10^{-6}$ per reactor, and thus of the order of $n 10^{-6}$ for an individual concerned with $n$ reactors in his/her neighbourhood. Similarly, major earthquakes in the United-States affect individuals homeowners with low probabilities. According to the United States Geological Survey, the probability of a major earthquake with a magnitude of at least 7 in the San Francisco area in a 30 year period of time between 2014 and 2044 is 51\%. On an annual basis, this translates into a $2.35 \%$ probability. Assuming that such an earthquake affects $10 \%$ of the population in the San Francisco region leads to an individual probability of loss around $2 \%$.

Other risks such as large-scale terrorism or major sanitary crisis have very low probabilities but consequences severe enough to mobilise authorities. In the US, the Californian Earthquake Authority or the Terrorism Insurance Act are evidence of such a willingness to improve the coverage for these very low probability risks. In Europe, the Paris and Vienna conventions provide for a coverage against nuclear catastrophes financed jointly by the operators, and the states. The revision of these conventions in 2004 has lead to an increase in coverage but the fact that nuclear liability insurance remains insufficient is still the object of active policy debates.

The role that governments play in organising the coverage for such catastrophic risks is indicative of the difficulty to increase take-up in market environments for low-probability risks. To circumvent issues of low take-up rates, countries like France or Spain have even adopted a fully compulsory natural disaster insurance scheme.

Despite their low probabilities, such events are costly to insure, in particular because they affect many individuals simultaneously and require that insurers have access to important sources of financing in case of accident. 
This cost, passed on to consumers may discourage insurance take-up. The goal of this paper is to highlight the conditions under which a very low probability catastrophe remains nevertheless insurable when capital is costly.

Figure 1 shows the evolution of the share of insured losses to total economic losses from natural disasters in the world from 1980 to 2018. This ratio has a positive trend but remains limited. Even in the most recent years it remained below $50 \%$ at $144 / 345 \approx 47 \%$ in 2018 and $80 / 170 \approx 50 \%$ in 2017. These average coverage rates hide important disparities between countries, and insurance penetration is much higher in high-income countries, ${ }^{1}$ where $56 \%$ of the natural catastrophe losses were insured in 2018, than in low-income ones, where virtually none of the natural catastrophe losses were insured. Nevertheless even in high-income countries, the average coverage rate for the period 2000-2018 is $46 \%$ with substantial heterogeneity between individuals. For instance, only $40 \%$ of the residents in New-Orleans had flood insurance when Hurricane Katrina hit in 2005 and many victims of Hurricane Sandy in 2012 had no flood insurance at all.

Our perspective will mainly be normative. In practice, there are many reasons why catastrophic risks often remain poorly insured, as shown by an important body of literature following Kunreuther (1973). The explanations for these low coverage rates have ranged from departures from the expected utility paradigm (Kunreuther \& Slovic (1978), Kunreuther et al. (2001) and Hertwig et al. (2004)) to the crowding-out of private insurance demand by public relief (Raschky et al. (2013), Kousky et al. (2013) and Grislain-Letrémy (2018)). Imperfect capital markets are also often presented as a significant impediment to the insurability of catastrophic risks (Jaffee \& Russell (1997), Froot (2001), Niehaus (2002), Zanjani (2002), Cummins et al. (2002) and Kousky \& Cooke (2012)).

If market failures and behavioural biases are needed to justify government interventions, they are not necessary a priori to rationalise low insurance take-up rates. As we will show, covering risks that have a systemic component is, by nature, particularly costly, even in a perfectly competitive set-up. Disentangling the role of market failures and behavioural biases from the intrinsic characteristics of the risk that may make them uninsurable is therefore an important pre-requisiste to relevant policy intervention. Put differently, it is important to understand if low-probability high-severity risks may not be adequately covered because of imperfections in market organisations and/or behavioural biases in decision-making, or if they are by nature, uninsurable.

\footnotetext{
${ }^{1}$ The classification follows Munich Re NatCat Service and is based on the World Bank's Gross National Income (GNI) measure. Countries with GNI below 1,045 US dollars are classified as low-income and countries with GNI above 12,736 US dollars are classified as high-income. Other countries are considered medium-income.
} 


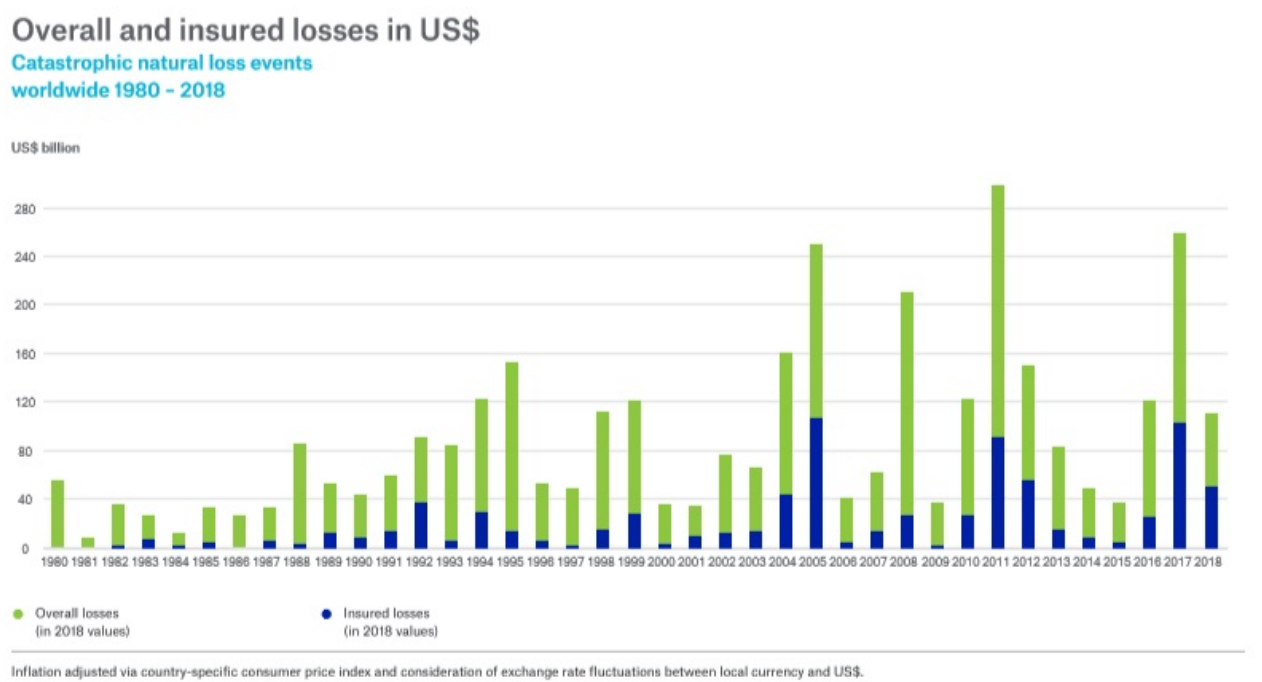

Figure 1: Evolution of economic losses and insured losses from natural catastrophes worldwide (Earthquakes - Tsunamis - Convective storms - Tropical cyclones - Winter storms - Winter damages - Flood - Flask floods - Heat waves - Wild fires) from 1980 to 2019. Source : (C) 2019 Munich Re, Geo Risks Research, NatCatSERVICE. As of November 2019.

The present paper consequently remains upstream of market failures and departures from expected utility, and our objective is to highlight the intrinsic determinants of demand and supply in the insurance market for catastrophic risks. To some extent, this does not differ from a standard market analysis, where explanatory factors determine demand and supply. However, as we will see, the insurance coverage of events that are, at the same time, very rare (low probability) and simultaneously highly damaging (high severity) to many people (correlated) requires a specific approach. On the demand side, the severity of the losses incurred by individuals makes insurance particularly valuable, but low probabilities reduce the propensity of individuals to purchase insurance. The willingness to pay for the coverage of such risks results from these counter-balancing effects. On the supply side, the fact that many individuals are affected at the same time when a disaster occurs makes insurance costlier to provide, since capital must be raised to maintain the insurer's solvency.

Our objective in this paper is to analyse these specificities, in order to bring out the key determinants of insurance coverage for catastrophic risks. In a nutshell, it will turn-out that these key determinants are the degree of risk aversion that individuals feel when they experience the highest possible 
loss and the correlation between individual risks.

Our analysis shows that, in a perfectly competitive insurance market without excessive transaction costs, low-probability risks that entail serious losses for victims are (at least partially) covered, in spite of their systemic nature. A contrario, and from a positive economics perspective, this suggests that substantial market failures, behavioural biases or transaction costs are likely to play a key role in the explanation of low catastrophic insurance take-up rates observed in practice. From a normative standpoint, our analysis emphasise the favourable welfare effects of policies that seek to organise insurance schemes against catastrophic risks by providing information on risks, by lowering transaction costs or by mitigating the various forms of market failures that may be at stake.

As a preliminary step, we leave the correlation aspect of catastrophic risks out of the picture to focus on how low-probability, high-severity risks can be viewed through the lens of insurance microeconomics. To do so, we first move away from the Arrow-Pratt approximation Arrow (1963) and Pratt (1964)) of the risk premium in order to account for potentially large deviations from the mean, and we provide an asymptotic characterisation of the willingness to pay to get rid of a very low-probability risks. We show that, when individuals display decreasing absolute risk aversion (DARA), a high absolute risk aversion (or, equivalently, a low risk tolerance) in the accident state may entail a significant willingness to pay to avoid risk, even if the accident probability is very low (an extreme case being infinite absolute risk aversion in the loss state, as in Weitzman (2009)).

We then investigate the optimal insurance coverage of an individual who faces the risk of an accident with a very low probability. We extend the canonical model of the optimal insurance literature (Mossin (1968) and Raviv (1979)) by considering a general insurance pricing rule reflecting the increasing marginal cost of capital, and we characterise the asymptotic optimal insurance coverage when the loss probability tends to zero.

Considering an increase in the size of a potential loss and a decrease in the probability of such a loss is reminiscent from an important body of literature that addresses the effect of a change in risk on optimal individual choices, in particular the demand for risky assets. Rothschild \& Stiglitz (1971) have highlighted the conditions under which an increase in risk lowers expected utility, and Gollier (1995) found the necessary and sufficient conditions under which an increase in risk leads to an increase in the demand for a risky asset by a risk averse individual. Our approach is different and complementary to the latter. We do not consider the effect of a second-order stochastic dominance shift of the risk distribution associated to a simultaneous decrease in the probability and increase in the severity of an accident. Instead we con- 
sider that an accident (the catastrophe) may trigger a (presumably severe) loss, whose distribution is fixed, and we analyse insurance demand when the probability of occurrence of the accident becomes very small. This approach allows us to characterise insurance demand for any catastrophe whose probability of occurrence is sufficiently close to zero. Of course, the scope of this asymptotic is limited to risks whose probability of occurrence is sufficiently close to zero. Its relevance to address actual examples of catastrophic risks is therefore investigated in a subsequent numerical example with realistic assumptions on preferences, risk distribution and costs of insurance.

We then study the supply side of the insurance market by considering the risk of a low-probability catastrophe affecting a large number of individuals at the same time, hence acknowledging the correlation aspect of catastrophic risks. When the catastrophe takes place, we assume that a random fraction of the population is affected by the loss. The higher the variance of this random variable, the higher the volatility of the insurer's liability. The model allows to span a continuum of situations between perfect correlation, when either no-one is affected or everybody is, and no correlation, when the same fraction of individuals always incurs the loss. To maintain its solvency, the insurance provider raises capital on financial markets and, since the catastrophe is systemic, capital is costly to obtain and features a positive risk premium. This cost of capital is passed onto the policyholders through the insurance premiums, that are therefore above the actuarially fair prices. Low levels of coverage may consequently occur in equilibrium when the catastrophe is highly systemic, even in a complete financial market setting. We show however that the optimal insurance coverage remains positive for low-probability risks.

Our conclusions may also be presented in a more normative perspective, by focusing on their policy implications. Removing the barriers to catastrophic risk insurability is a multi-faceted challenge, including the promotion of adequate financial innovations, and the targeting of government policy towards risk prevention and the assistance of the most vulnerable groups. Our approach is restricted to the preliminary question as to whether, and under which conditions, the coverage of catastrophic risks by insurance mechanisms is a socially optimal objective. The answer to this question is positive if the individual's degree of absolute risk aversion, evaluated in the accident state, overcompensates the high price of insurance induced by the systemic nature of the risk. We will show that this condition is satisfied if insurance transaction costs are not too large. Reducing transaction costs (e.g., by lowering distribution and claim handling costs) is thus a crucial step to- 
ward insurability $\left.\right|^{2}$ Put differently, when transaction costs could be reduced by improving market organisations, assuming as a matter of principle, that individuals should be protected by state-sponsored insurance mechanisms, whatever their willingness to pay and the cost of capital, does not seem to be the appropriate way to approach the catastrophic risk insurance issue.

Finally, we complete these theoretical foundations by simulating the optimal insurance coverage. Our simulations yield significant levels of coverage. We also show that the optimal coverage converges rapidly to its limit, which corroborates the relevance of the asymptotic approach used in the previous sections.

The rest of the paper is organised as follows. Section 2 and 3 respectively analyse the risk premium and the insurance demand for a low-probability, high-severity risk, and Section 4 provides the analysis of the supply side of insurance, taking the issue of risk correlation into account. Section 5 illustrates the relevance of our results with numerical simulations and Section 6 concludes by summarising our findings and relating them to public policy issues. Section 7 gathers the proofs.

\section{The risk premium of low-probability high- severity risks}

The Arrow-Pratt approximation of the risk premium holds for small risks, with little variation around the mean. As a preliminary analysis of our study of optimal insurance against catastrophic risks, this section characterises the risk premium of low-probability, high-severity risks.

Consider a risk-averse individual with a (twice continuously differentiable) von Neumann-Morgenstern utility function $u(x)$ such that $u^{\prime}>0$ and $u^{\prime \prime}<$ 0 , where $x \geq 0$ is the individual's wealth. Let $A(x)=-u^{\prime \prime}(x) / u^{\prime}(x)$ and $T(x)=1 / A(x)$ be his indices of absolute risk aversion and of risk tolerance, respectively. He holds an initial wealth $w$, and faces the risk of a loss $\tilde{\ell}$, which may occur with probability $p$. Hence $p$ is the probability of an event (the "accident", or "catastrophe") that triggers the random loss $\tilde{\ell}$. Conditionally on the occurrence of an accident, $\tilde{\ell}$ is distributed in $[0, \bar{L}]$, with c.d.f. $F(\ell)$ and density $f(\ell)=F^{\prime}(\ell)$ and $\bar{L} \leq w$. Thus $m(p, \tilde{\ell})=p \mathbb{E} \tilde{\ell}$ and $\sigma^{2}(p, \tilde{\ell})=$ $p(1-p)(\mathbb{E} \tilde{\ell})^{2}+p \sigma_{\ell}^{2}$, where $\sigma_{\ell}^{2}=\operatorname{Var}(\tilde{\ell})$ are, respectively, the expected value and the variance of the loss. The certainty equivalent $C(p, \tilde{\ell})$ of lottery $(p, \tilde{\ell})$

\footnotetext{
${ }^{2}$ Important aspects of the US N.F.I.P. are illustrative of this approach, by facilitating the underwriting of flood policies, (e.g., the "write your own policy"), and through ground intervention of F.E.M.A agents in the case of a flood.
} 
is defined by

$$
u(w-C)=(1-p) u(w)+p \mathbb{E} u(w-\tilde{\ell}) .
$$

Straightforward calculations give

$$
\begin{aligned}
C_{p}^{\prime}(p, \tilde{\ell}) & =\frac{u(w)-\mathbb{E} u(w-\tilde{\ell})}{u^{\prime}(w-C)}>0, \\
C_{p^{2}}^{\prime \prime}(p, \tilde{\ell}) & =-C_{p}^{\prime}(p, \tilde{\ell})^{2} A(w-C)<0 .
\end{aligned}
$$

Thus, $C(p, \tilde{\ell})$ is increasing and concave with respect to $p$, and of course we have $C(0, \tilde{\ell})=0$. We also denote

$$
\theta(p, \tilde{\ell}) \equiv \frac{C(p, \tilde{\ell})-m(p, \tilde{\ell})}{\sigma^{2}(p, \tilde{\ell})},
$$

the normalised risk premium, that is the risk premium per unit of variance.

Put informally, the risk $(p, \tilde{\ell})$ may be considered catastrophic for the individual if $C(p, \tilde{\ell})$ is non-negligible although $p$ is very small. Risk aversion implies that $C(p, \tilde{\ell})>p \mathbb{E} \tilde{\ell}$. Denoting $C_{p}^{\prime}\left(0_{+}, \tilde{\ell}\right) \equiv \lim _{p \rightarrow 0} C_{p}^{\prime}(p, \tilde{\ell})$, L'Hôpital's rule allows us to write the limit ratio of certainty equivalent to expected loss as

$$
\lim _{p \rightarrow 0} \frac{C(p, \tilde{\ell})}{m(p, \tilde{\ell})}=\frac{C_{p}^{\prime}\left(0_{+}, \tilde{\ell}\right)}{\mathbb{E} \tilde{\ell}},
$$

which is proportional to $C_{p}^{\prime}\left(0_{+}, \tilde{\ell}\right)$ for $\tilde{\ell}$ given. Using l'Hôpital's rule again gives

$$
\theta\left(0_{+}, \tilde{\ell}\right) \equiv \lim _{p \longrightarrow 0} \theta(p, \tilde{\ell})=\frac{C_{p}^{\prime}\left(0_{+}, \tilde{\ell}\right)-\mathbb{E} \tilde{\ell}}{(\mathbb{E} \tilde{\ell})^{2}}
$$

Thus, analysing the determinants of $\theta\left(0_{+}, \tilde{\ell}\right)$ is an intermediate step to understanding why $C_{p}^{\prime}\left(0_{+}, \tilde{\ell}\right)$ may be large and thus why $C(p, \tilde{\ell})$ may be significant although $p$ is very small.

When $\tilde{\ell}$ is small, we know from the Arrow-Pratt approximation that the risk premium per unit of variance is proportional to the index of absolute risk aversion. Indeed, for $p$ fixed, considering a sequence of random variables $\tilde{\ell}_{n}$ with support $\left[0, \bar{L}_{n}\right]$ and $\bar{L}_{n} \rightarrow 0$ when $n \rightarrow+\infty$ (hence with $\mathbb{E} \tilde{\ell}_{n} \rightarrow 0$ and $\operatorname{Var} \tilde{\ell}_{n} \rightarrow 0$ when $\left.n \rightarrow+\infty\right)$, we have

$$
\lim _{n \rightarrow+\infty} \theta\left(p, \tilde{\ell}_{n}\right)=\frac{A(w)}{2} \text { for all } p \in(0,1),
$$

which of course also holds when $p$ goes to 0 , that is

$$
\lim _{n \longrightarrow+\infty} \theta\left(0_{+}, \tilde{\ell}_{n}\right)=\frac{A(w)}{2} .
$$


When $\tilde{\ell}$ can take large values, it is intuitive that the size of the risk premium depends on function $A(x)$ not only in the neighbourhood of $x=w$, but over the whole interval $[w-\bar{L}, w]$. This is confirmed by Proposition 1 and its corollary. Proposition 1 provides an exact formula for $\theta\left(0_{+}, \tilde{\ell}\right)$ which is a weighted average of $A(x) \exp \left\{\int_{x}^{w} A(t) d t\right\} / 2$ when $x$ is in $[w-\bar{L}, w]$ and Corollary 1 directly deduces a lower bound for $\theta\left(0_{+}, \tilde{\ell}\right)$.

Proposition 1. For all $\tilde{\ell}$, the asymptotic normalised risk premium is

$$
\theta\left(0_{+}, \tilde{\ell}\right)=\frac{1}{2} \int_{w-\bar{L}}^{w}\left[k(x) A(x) \exp \left\{\int_{x}^{w} A(t) d t\right\}\right] d x
$$

where

$$
k(x)=\frac{2\left[x-(w-\bar{L})-\int_{w-x}^{\bar{L}} F(\ell) d \ell\right]}{(\mathbb{E} \tilde{\ell})^{2}}>0
$$

and

$$
\int_{w-\bar{L}}^{w} k(x) d x=1
$$

Corollary 1. For all $\tilde{\ell}$, a lower bound for the asymptotic normalised risk premium is given by

$$
\theta\left(0_{+}, \tilde{\ell}\right)>\frac{1}{2} \int_{w-\bar{L}}^{w} k(x) A(x) d x .
$$

With the natural case of non-increasing absolute risk aversion in mind, Proposition 1 and Corollary 1 suggest that $\theta\left(0_{+}, \tilde{\ell}\right)$ may be large if $A(x)$ is large (or equivalently when $T(x)$ is low) when $x$ goes to $w-\bar{L}$.

Symmetrically, Proposition 2 and Corollary 2 show that, under nonincreasing absolute risk aversion, the normalised risk premium $\theta(p, \tilde{\ell})$ may be large when $p$ is close to zero only if $A(w-\bar{L})$ is very large, that is, only when the individual's risk tolerance is very small in the accident state.

Proposition 2. Let

$$
\varepsilon(x)=\frac{x[1-F(w-x)]}{\int_{w-x}^{\bar{L}}[1-F(\ell)] d \ell}
$$

and

$$
\bar{\gamma}=\max \{R(x), x \in[w-\bar{L}, w]\} .
$$

Assume $\bar{L}<w, \bar{\gamma}<w / \mathbb{E} \tilde{\ell}, f(\bar{L})>0, f^{\prime}(\bar{L})<0$ and $\varepsilon(x)$ is decreasing over $[w-\bar{L}, w]$. Then, we have

$$
\theta\left(0_{+}, \tilde{\ell}\right)<\frac{\bar{L}}{\mathbb{E} \tilde{\ell}} \bar{A}
$$


where $\bar{A}=1 / \bar{L} \int_{w-\bar{L}}^{w} A(x) d x$, and

$$
C(p, \tilde{\ell})<p \mathbb{E} \tilde{\ell}[1+\bar{L} \bar{A}]
$$

Corollary 2. In addition to the assumptions made in Proposition 2, under non-increasing absolute risk aversion, we have

$$
\theta\left(0_{+}, \tilde{\ell}\right)<\frac{\bar{L}}{\mathbb{E} \tilde{\ell}} A(w-\bar{L})
$$

and

$$
C(p, \tilde{\ell})<p \mathbb{E} \tilde{\ell}[1+\bar{L} A(w-\bar{L})]
$$

Proposition 2 provides upper bounds for the asymptotic normalised risk premium $\theta\left(0_{+}, \tilde{\ell}\right)$ and for the certainty equivalent $C(p, \tilde{\ell}) . \bar{\gamma}$ is an upper bound for the index of relative risk aversion $R(x)$ when $x$ is in the interval $[w-\bar{L}, w]]^{3}$ The upper bound of $\theta\left(0_{+}, \tilde{\ell}\right)$ and $C(p, \tilde{\ell})$ are proportional to the average index of absolute risk aversion $\bar{A}$. Corollary 2 focuses on the nonincreasing absolute risk aversion case and shows that $\theta\left(0_{+}, \tilde{\ell}\right)$ and $C(p, \tilde{\ell})$ may be non-negligible when $p$ is very small, say as a proportion of the expected loss $\mathbb{E} \tilde{\ell}$, only if $A(w-\bar{L})$ is large. On the contrary, assume $A(w-\bar{L})=A(w)$, i.e., the index of absolute risk aversion remains constant in $[w-\bar{L}, w]$. In that case, we would have $R(x)<R(w)$ for all $x<w$, and thus $\bar{\gamma}=R(w)$, which implies

$$
\begin{aligned}
C(p, \tilde{\ell}) & <p \mathbb{E} \tilde{\ell}[1+\bar{L} A(w)] \\
& =p \mathbb{E} \tilde{\ell}\left(1+\frac{\bar{L}}{w} \bar{\gamma}\right)
\end{aligned}
$$

Assuming $R(w)=2$ or 3 and $\bar{L}=\theta w$ with $\theta \in[0,1[$ would give $C(p, \tilde{\ell})<$ $p \mathbb{E} \tilde{\ell}(1+2 \theta)$ or $C(p, \tilde{\ell})<p \mathbb{E} \tilde{\ell}(1+3 \theta)$, respectively. Thus, if $p$ is very small, then $C(p, \tilde{\ell}) / \mathbb{E} \tilde{\ell}$ is very small. $\tilde{4}^{4}$

\footnotetext{
${ }^{3}$ We have $\varepsilon(w)=w / \mathbb{E} \tilde{\ell}$ and $\varepsilon(x) \rightarrow+\infty$ when $x \rightarrow w-\bar{L}$. Hence the monotonicity assumption made in the proposition guarantees $\varepsilon(x)>w / \mathbb{E} \tilde{\ell}$ for all $x$. If this monotonicity assumption is not made, Proposition 2 holds under $\bar{\gamma}<\min \{\varepsilon(x), x \in[w-\bar{L}, w]\}$.

${ }^{4}$ For the sake of numerical illustration, consider the case of a large-scale nuclear disaster that may occur with probability $p=10^{-5}$, with expected total losses of $\$ 100 \mathrm{~b}$ evenly spread among 1 million inhabitants (think of people living in the neighbourhood of the nuclear plant). In the case of an accident, each inhabitant would suffer a loss of expected value $\mathbb{E} \tilde{\ell}=\$ 100,000$. The unconditional expected loss $p \mathbb{E} \tilde{\ell}$ equals $\$ 1$, and the certainty equivalent is less than $1+2 \theta<\$ 3$ or $1+3 \theta<\$ 4$, which is negligible, say as a proportion of their annual electricity expenses. Assuming larger but still realistic values of the index of relative risk aversion would not substantially affect this conclusion. If we assume $w=\$ 2 M$, then the condition $\bar{\gamma} \leq w / \mathbb{E} \tilde{\ell}$ is written as $\bar{\gamma} \leq 20$.
} 
Thus, under non-increasing absolute risk aversion, we may conclude that the risk premium of low-probability high-severity accidents may be nonnegligible (and thus that the coverage of such a risk is a relevant issue) if and only if the risk tolerance is very low in such catastrophic cases.

$C R R A$ preferences are an instance of such a case with $T(x)=x / \gamma$, where $\gamma$ is the index of relative risk aversion. We then have $T(x) \longrightarrow 0$ and $A(x) \longrightarrow \infty$ when $x \longrightarrow 0$. However, $C R R A$ preferences are not very well suited to deal with large risks because the utility function has an explosive behaviour when final wealth approaches zero. As a consequence, considering large losses can yield results extremely sensitive to even reasonable variations in the level of risk aversion 5

If preferences are of the $H A R A$ type, the utility function is written as

$$
u(x)=\zeta\left(\eta+\frac{x}{\gamma}\right)^{1-\gamma},
$$

whose domain is such that $\eta+(x / \gamma)>0$, and with the condition $\zeta(1-\gamma) / \gamma>$ 0 , that guarantees that $u(x)$ is increasing and concave. HARA preferences correspond to affine risk tolerance $T(x)=1 / A(x)=\eta+x / \gamma$ and the class nests the constant relative risk aversion (CRRA) case when $\eta=0$, and the constant absolute risk aversion (CARA) case when $\gamma \rightarrow+\infty$. Keeping the DARA case in mind leads to the additional restriction $\gamma>0$.

When $\tilde{\ell}$ is close to a Dirac distribution at $\ell=w$, i.e. when the individual is at risk of loosing close to $w$ in case of a catastrophe, it can be shown that ${ }^{6}$

$$
\theta\left(0_{+}, \tilde{\ell}\right)>\frac{1}{w} \int_{0}^{w} \frac{x u^{\prime}(x)}{w u^{\prime}(w)} A(x) d x
$$

which gives

$$
\theta\left(0_{+}, \tilde{\ell}\right)>\frac{\gamma}{w^{2}} \int_{0}^{w} \frac{x}{\eta \gamma+x}\left(\frac{\eta \gamma+w}{\eta \gamma+x}\right)^{\gamma} d x \equiv \Gamma(\eta)
$$

A short computation shows that if and only if $\gamma<1$. Otherwise, when $\gamma>1$ we have

$$
\lim _{\eta \rightarrow 0} \Gamma(\eta)=+\infty
$$

${ }^{5}$ See Eeckhoudt et al. (2000) and Weitzman (2009) for examples of this sensitivity issue, and Ikefuji et al. (2015) for a formal analysis of the conditions under which the problem arises.

${ }^{6}$ This can be obtained either by using equation 23 in the proof of Proposition 1 with $F(\ell)=0$ if $\ell<\bar{L}$ and $F(\ell)=1$ if $\ell>\bar{L}$, or equivalently, by directly establishing Proposition 1 in this simpler case. 
when $\gamma>1$. The normalised risk premium $\theta\left(0_{+}, \tilde{\ell}\right)$ can therefore be made arbitrarily large by choosing a value $\eta=T(0)$ small enough, for $\gamma>1.7$

\section{Insurance demand for catastrophic risks}

We now assume that the individual can purchase insurance for a binomial risk $(p, L)$, where $\tilde{\ell}=L$ with probability 1 . Insurance contracts specify the indemnity $I$ in the case of an accident, i.e., when the individual suffers a loss $L$, and the premium $D(p, I)$ to be paid to the insurer depends on the loss probability $p$ and the indemnity $I$. As an example, the standard insurance pricing model specifies a price proportional to the expected indemnity $D(p, I)=(1+\lambda) p I$, where $\lambda$ is the loading factor. More generally, we call

$$
d(p, I)=\frac{D(p, I)-p I}{p I},
$$

the unit cost of insurance with $d(p, I)=\lambda$ in the case of a constant loading. In the case of catastrophe risk with correlated claims however, $d(p, I)$ is strongly affected by the cost of providing the capital that guarantees the solvency of the insurance scheme and it is likely to depend on $p$ and $I$. We assume non-decreasing marginal costs $D_{I^{2}}^{\prime \prime}(p, I) \geq 0$, and $D_{I}^{\prime}(p, I) \in(p, 1)$ rules out corner solutions with $I=0$ or $I=L$. We also assume that the marginal cost of coverage becomes null as $p$ tends to zero, that is

$$
\lim _{p \rightarrow 0} D_{I}^{\prime}(p, I) \equiv D_{I}^{\prime}\left(0_{+}, I\right)=0
$$

and consistency conditions $D(p, 0)=D\left(0_{+}, I\right)=0$.

The policyholder faces the lottery $\left(w_{1}, w_{2}\right)$, with corresponding probabilities $1-p$ and $p$, where $w_{1}$ and $w_{2}$ denote the wealth in the no-loss and loss states respectively, with

$$
\begin{array}{r}
w_{1}=w-D(p, I) \\
w_{2}=w-D(p, I)-L+I
\end{array}
$$

The full coverage lottery $w_{1}=w_{2}=w-D(p, L)$ is preferred to the no coverage lottery $(w, w-L)$ if and only if the certainty equivalent, now denoted $C(p, L)$ is higher than the price of full coverage $D(p, L)$, that is

$$
C(p, L) \geq D(p, L) .
$$

\footnotetext{
${ }^{7}$ Note that $R(x)$ is increasing in the HARA case, and assuming $R(x)>1$ when $x$ is large (e.g. when $x$ is close to $w$ ) requires $\gamma>1$.
} 
When $p$ goes to zero, l'Hôpital's rule allows us to rewrite the previous condition as

$$
\frac{C_{p}^{\prime}\left(0_{+}, L\right)-L}{L}=\theta\left(0_{+}, L\right) L \geq d\left(0_{+}, L\right),
$$

where $d\left(0_{+}, I\right)=\lim _{p \rightarrow 0} d(p, I)$. Hence the following Lemma.

Lemma 1. $\theta\left(0_{+}, L\right) L \geq d\left(0_{+}, L\right)$ is a necessary and sufficient condition for the individual to prefer full insurance to no insurance when $p$ goes to zero.

Lemma 1 illustrates the importance of the normalised risk premium $\theta\left(0_{+}, L\right)$ analysed in the previous section. For insurance to remain attractive despite the vanishingly low probability of accident, the normalised risk premium has to be larger than the unit cost of insurance $d\left(0_{+}, L\right)$ divided by the loss. A direct consequence of Lemma 1 is that $\theta\left(0_{+}, L\right) L \geq d\left(0_{+}, L\right)$ is a sufficient condition for the optimal (partial) insurance cover to remain positive as $p$ goes to zero. 8

Combining Corollary 1 and Lemma 1 yields Corollary 3 that provides conditions on relative risk aversion under which insurance remains valuable for low-probability events with high severity.

Corollary 3. Assume $R(x)$ is non decreasing. If $\lim _{x \rightarrow 0} R(x) \geq \lim _{L \rightarrow w} d\left(0_{+}, L\right)$, then for $L$ smaller than $w$ but large enough, the individual prefers full insurance to no insurance (and therefore the optimal cover is positive) when $p$ goes to zero.

Decreasing the probability and increasing the loss of the accident while maintaining the expected loss is equivalent to a mean-preserving spread. As is well known, this induces a decrease in the expected utility of a riskaverse individual. The amount of money that such an individual would be willing to pay to fully cover such a risk would therefore increase. Corollary 3 provides an additional insight : if individuals have a coefficient of relative risk aversion in the worst case scenario higher than the price of coverage, then they will prefer full coverage over no coverage for any infinitesimally small probability accident. For example, with the standard proportional loading rule $D(p, I)=(1+\lambda) p I$ and a CRRA utility function with relative risk aversion coefficient $\gamma$, the condition of Corollary 3 simply is written as $\gamma>\lambda$.

Let us now characterise the optimal insurance coverage for a low-probability accident. In the $\left(w_{1}, w_{2}\right)$ plane represented in Figure 2, the set of feasible

\footnotetext{
${ }^{8}$ Indeed, if the individual prefers full coverage to no coverage, extending his opportunity set does not make him switch to zero coverage. It is easy to check that the optimal limit cover (denoted $I^{*}$ below) is positive when $d\left(0_{+}, L\right)<\left[u^{\prime}(w-L)-u^{\prime}(w)\right] / u^{\prime}(w)$ and that this condition is implied by $\theta\left(0_{+}, L\right) L \geq d\left(0_{+}, L\right)$.
} 
lotteries is delimited by a concave curve (drawn for $p=0.1, p=0.25$ and $p=0.5)$ that represents equations (4) and (5), together with the sign condition

$$
w_{2}-w_{1}+L \geq 0,
$$

(or $I \geq 0$ ). For illustrative purpose, Figure 2 displays the case of a simple pricing rule that relates the premium to the expected value and variance of the loss..$^{9}$ The optimal lottery maximizes the individual's expected utility

$$
(1-p) u\left(w_{1}\right)+p u\left(w_{2}\right)
$$

in this set of feasible lotteries. It is such that the marginal rate of substitution $-d w_{2} / d w_{1 \mid E u=c t .}=(1-p) u^{\prime}\left(w_{1}\right) / p u^{\prime}\left(w_{2}\right)$ is equal to the slope (in absolute value) of the feasible lottery locus, that is

$$
(1-p) D_{I}^{\prime}(p, I) u^{\prime}\left(w_{1}\right)=\left[1-D_{I}^{\prime}(p, I)\right] p u^{\prime}\left(w_{2}\right),
$$

where $w_{1}$ and $w_{2}$ depend on $I$ through (4) and (5). Figure 2 shows the locus of optimal lotteries in the $\left(w_{1}, w_{2}\right)$ plane when $p$ changes. Each lottery is at the tangency point of a convex indifference curve with the concave curve that delimits the set of feasible lotteries for a particular probability $p$. Point A represents the situation with no insurance, and point B represents the asymptotic optimal lottery when $p$ goes to zero.

Let $w_{1}(p, L), w_{2}(p, L)$ denote the optimal state-contingent wealth levels when $I>0$, that is, when $d(p, I)$ is not too large. Equation (7) can be rewritten as

$$
\frac{u^{\prime}\left(w_{2}(p, L)\right)}{u^{\prime}\left(w_{1}(p, L)\right)}=\frac{(1-p) D_{I}^{\prime}(p, I)}{p\left(1-D_{I}^{\prime}(p, I)\right)} .
$$

Denoting

$$
\begin{aligned}
w_{1}^{*}(L) & \equiv \lim _{p \longrightarrow 0} w_{1}(p, L)=w \\
w_{2}^{*}(L) & \equiv \lim _{p \longrightarrow 0} w_{2}(p, L),
\end{aligned}
$$

and using L'Hôpital's rule yields

$$
u^{\prime}\left(w_{2}^{*}(L)\right)=D_{I p}^{\prime \prime}\left(0_{+}, I^{*}\right) u^{\prime}\left(w_{1}^{*}(L)\right),
$$

which implies $w_{2}^{*}(L)<w=w_{1}^{*}(L)$ if and only if $D_{I p}^{\prime \prime}\left(0_{+}, I^{*}\right)>1$. Thus, when $p$ goes to 0 , the optimal insurance contract $(P, I)$ goes to a limit $\left(P^{*}, I^{*}\right)$, with $P^{*}=D\left(0_{+}, I^{*}\right)=0$ and $I^{*}=w_{2}^{*}(L)+L-w_{1}^{*}(L)<L$. When $p$ is

\footnotetext{
${ }^{9}$ Section 4 will be dedicated to analyzing the supply side of the market and will derive a pricing function.
} 
Figure 2: Comparative statics in the space of lotteries

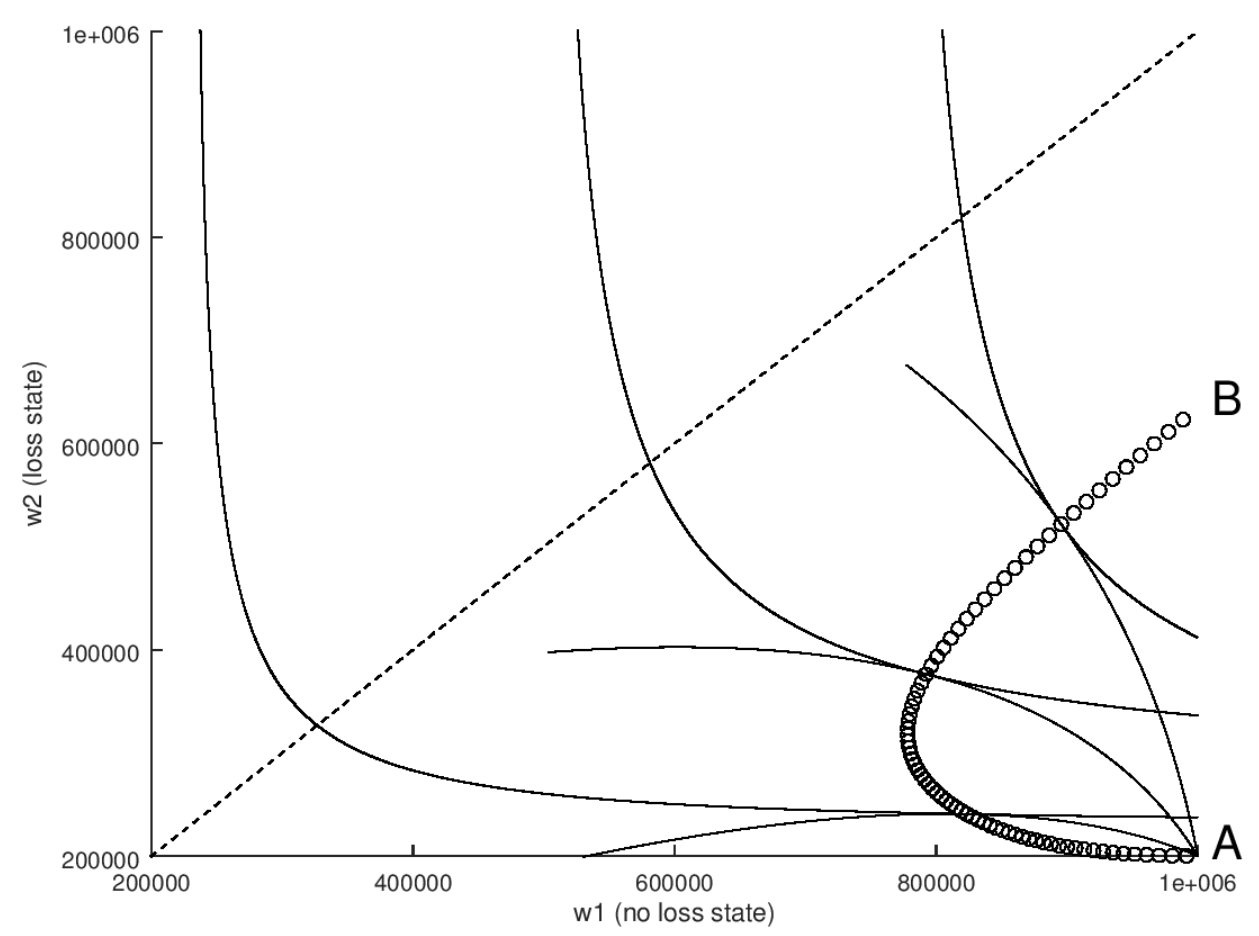

Each thick black circle represents an optimal lottery for a given probability, from $p$ high in point A to $p$ close to zero in point B. Optimal lotteries are at the tangency point of the indifference curve and the cost curve. In $\mathrm{B}$, the optimal insurance coverage is positive even-though the loss probability is infinitesimally small. The calibration is $w=1,000,000$, $L=800,000, u(x)=-\frac{x^{-2}}{2}$, and $D(p, I)=(1+\lambda) p I+\alpha p(1-p) I^{2}$ with $\lambda=0.3$ and $\alpha=3 \times 10^{-6}$.

positive but close to 0 , we still have $I<L$ and $P=D(p, I) \simeq D\left(p, I^{*}\right)$. Since $w_{2}^{*}(L)=w-L+I^{*},(8)$ gives

$$
u^{\prime}\left(w-L+I^{*}\right)=D_{I p}^{\prime \prime}\left(0_{+}, I^{*}\right) u^{\prime}(w)
$$

which defines $I^{*}$ when $I^{*}>0$, that is when $u^{\prime}(w-L)>D_{I p}^{\prime \prime}\left(0_{+}, 0_{+}\right) u^{\prime}(w)$, where $D_{I p}^{\prime \prime}\left(0_{+}, 0_{+}\right)=\lim _{p, I \rightarrow 0} D(p, I)$. Otherwise, we have $I^{*}=0$.

Proposition 3. Assume that $D_{I p}^{\prime \prime}\left(0_{+}, 0_{+}\right) \leq \frac{u^{\prime}(w-L)}{u^{\prime}(w)}$. Then, when $p$ goes to 0 , the optimal insurance coverage $I$ goes to a limit $I^{*}>0$ defined by Equation (9), and when $p$ is close to $0, I$ and $P$ are close to $I^{*}$ and $D\left(p, I^{*}\right)$, respectively. 
Proposition 3 highlights the conditions under which a risk with an infinitesimally small probability remains insurable. For example, with a proportional loading, a logarithmic utility and a loss equal to a fraction $\theta$ of $w$ the conditions summarise as $1+\lambda<1 /(1-\theta)$. That is, the loading must be sufficiently small and the loss sufficiently large. The generality of Proposition 3 in terms of utility and cost functions will allow us to discuss the insurability of catastrophic risks in the next section.

Straightforward calculations allow us to characterise the effect of a change in $L$ and/or $w$ on the asymptotic optimal insurance coverage. An increase $d L>0$ for $w$ given induces an increase $d I^{*}<d L$. A simultaneous increase $d w=d L>0$ induces an increase $d I^{*}>0$ in coverage, while an increase in wealth with unchanged loss $d w>0, d L=0$ entails a decrease in optimal coverage $d I^{*}<0$ under $D A R A$ preferences, i.e. when $A^{\prime}<0$. These comparative statics results, standard in the analysis of traditional risks ${ }^{10}$ therefore extend to the asymptotic characterisation of catastrophic risk optimal insurance.

\section{Insurance supply for catastrophic risk}

This section determines the pricing rules $D(p, I)$ - taken as given in the previous section - and the corresponding insurance market equilibrium in an economy with a continuum of individuals and complete financial markets. To do so, we add a third component to the definition of catastrophic risks: in addition to their low-probability high-severity nature, they are correlated among individuals. Due to this correlation, many individuals may be simultaneously affected by the catastrophic event, hence its systemic nature.

Assume that a catastrophe occurs with probability $\pi$ and, in such a case, a fraction $\tilde{\kappa}$ of the population is affected by the loss $L$ (the same for all victims). In order to allow for different severity levels, we assume that $\tilde{\kappa}$ is a random variable with $\mathbb{E} \tilde{\kappa}=\mu_{\kappa}$ and $\operatorname{Var} \tilde{\kappa}=\sigma_{\kappa}^{2}$. Let

$$
\tilde{K}=\left\{\begin{array}{lll}
\tilde{\kappa} & \text { with probability } & \pi \\
0 & \text { with probability } 1-\pi
\end{array},\right.
$$

be the (unconditional) fraction of the population affected by the loss $L$. Conditionally on the realisation of $\tilde{K}$, we assume that all individuals have the same probability to incur the loss $L$. Therefore, the individual (unconditional) probability of facing a loss $L$ is $p=\pi \mu_{\kappa}$.

As can be anticipated, the variability of the average loss within the population will be a determinant of the insurance cost when this cost is transferred

\footnotetext{
${ }^{10}$ See Schlesinger (2013).
} 
to risk-averse investors. In the present setting, this variability is associated with a positive coefficient of correlation between individual losses which, as shown in Appendix 7.5, is equal to

$$
\rho=\frac{\sigma_{\kappa}^{2}+(1-\pi) \mu_{\kappa}^{2}}{\mu_{\kappa}-\pi \mu_{\kappa}^{2}} .
$$

The coefficient of correlation $\rho$ increases with $\sigma_{\kappa}^{2}$ and since $\tilde{\kappa}$ is distributed over the interval $[0,1]$, the highest variance is achieved when $\tilde{\kappa}$ is a Bernoulli variable, equal to 0 or 1 . The probability that $\tilde{\kappa}$ equals one in this case is simply $\mu_{\kappa}$, and we have $\sigma_{\kappa}^{2}=\mu_{\kappa}\left(1-\mu_{\kappa}\right)$, hence $\rho=1$. The highest possible variance $\sigma_{\kappa}^{2}$ therefore yields perfect correlation across losses ${ }^{11}$

Another knife-edge case arises when $\sigma_{\kappa}^{2}=0$ and $\pi=1$ that is, the same fraction of the population is always affected by the loss. This corresponds to the standard situation where the law of large number applies : the average loss is constant, and the correlation coefficient is $\rho=0$.

More generally, the correlation coefficient depends on the parameters $\mu_{\kappa}$, $\sigma_{\kappa}^{2}$ and $\pi$ and converges to

$$
\frac{\sigma_{\kappa}^{2}+\mu_{\kappa}^{2}}{\mu_{\kappa}}
$$

when $\pi \rightarrow 0$.

For a given probability distribution of $\tilde{K}$, the insurance provider offers a coverage $I$ at price $D$ to all agents in the economy. For each indemnity paid, the insurer incurs a proportional cost at rate $\lambda$ because of administrative expenses such as auditing and expertise, or other forms of transaction costs. Furthermore, in order to avoid a default, ${ }^{12}$ the insurance provider has to collateralise the random total indemnity costs $\tilde{K}(1+\lambda) I$. This may be achieved through various forms of contracting such as raising equity, in which case the insurance company's equity-holders are liable for the policy payments, or by raising capital on financial markets, in which case the risk is transferred to dedicated market investors. Formally, this is equivalent to assuming that the insurers collateralise indemnities by purchasing an asset that delivers a random payoff $\tilde{y}=\tilde{K}(1+\lambda) I$, whose price defines $D$.

In order to characterise this price, we consider a setting with complete financial markets, where all individuals have the same utility function $u$. The

\footnotetext{
${ }^{11}$ When $\pi>0$, this corresponds to the degenerate case where the catastrophe may be completely harmless. Only two events are then relevant : either there is a catastrophe that affects all individuals, either nobody suffers a loss, hence the perfect correlation.

${ }^{12}$ This paper abstracts from the possibility of default, examined in Charpentier \& Le Maux (2014) and Zanjani (2002) for example. In other words, we consider unlimited liability for all agents in the economy and in particular for the insurance provider.
} 
value of the asset $\tilde{y}$ can be estimated with a standard one factor mode ${ }^{13}$

$$
D=\mathbb{E} \tilde{y}+\operatorname{cov}\left(\tilde{y}, u^{\prime}(\tilde{z})\right) .
$$

where $\tilde{z}$ is the representative agent's random wealth (defined below). Under market completeness, the exposure of investors to idiosyncratic risks have been eliminated from the economy thanks to adequate contracting. As a consequence, only systemic risks give rise to a risk premium in equilibrium. The co-variance term of equation (12) captures the idea that investors, who accept to provide the necessary capital to sustain the insurance scheme, require a risk premium to provide a payoff which is high when the average wealth is low.

The representative agent has a wealth equal to the average wealth in the economy:

$$
\tilde{z}=w-\tilde{K} L,
$$

which is the difference between the exogeneously given level of initial wealth $w$ and the average loss per individual in the economy. A simple calculation using (10), 12 and 13 with $p=\pi \mu_{\kappa}$ and $\mathbb{E} \tilde{y}=p I$ gives

$$
D(p, I)=\psi(p) p I \quad \forall p \in\left[0, \mu_{\kappa}\right],
$$

where

$$
\psi(p)=(1+\lambda) \frac{\mathbb{E}\left[\tilde{\kappa} u^{\prime}(w-\tilde{\kappa} L)\right]}{\mu_{\kappa}}
$$

is the total loading that reflects both the transaction and capital costs of the insurance coverage, with utility normalisation $\mathbb{E} u^{\prime}(\tilde{z})=1 .{ }^{14}$

Since $p \rightarrow 0$ when $\pi \rightarrow 0$ with $\mu_{\kappa}$ given, the condition that guarantees that insurance take-up remains positive in the limit when $\pi$ tends to 0 can be derived from Proposition 3 as 5

$$
\psi(0) \leq u^{\prime}(w-L),
$$

\footnotetext{
${ }^{13}$ In an economy with complete financial markets, $D=\mathbb{E} \tilde{y}+\operatorname{cov}\left(\tilde{y}, \frac{u^{\prime}(\tilde{z})}{\mathbb{E} u^{\prime}(\tilde{z})}\right)$ defines the price of the asset $\tilde{y}$ (see Gollier (2004)). Without loss of generality, we use the normalisation $\mathbb{E} u^{\prime}(\tilde{z})=1$.

${ }^{14}$ Note that the right-hand side of 14 depends on $\pi, L$ and on the distribution of $\tilde{\kappa}$. In what follows, $L$ and $\tilde{\kappa}$ are considered as given and we analyse the effect of a change in the probability of a catastrophe $\pi$, that affects the individual probability $p=\mu_{\kappa} \pi$ of being a victim.

${ }^{15}$ When we consider economies that differ through the value of $\pi$, we maintain the normalising assumption $\mathbb{E} u^{\prime}(\tilde{z})=1$ for the economy. The utility functions therefore depend on $\pi$ and $u^{\prime}(w) \rightarrow 1$ when $\pi \rightarrow 0$ for any random variable $\tilde{\kappa}$.
} 
In addition, Equation (9) from the previous section also delivers a closedform solution for the optimal asymptotic coverage

$$
I^{*}=u^{\prime-1}(\psi(0))-w+L,
$$

where

$$
\psi(0)=(1+\lambda) \frac{\mathbb{E}\left[\tilde{\kappa} u^{\prime}(w-\tilde{\kappa} L)\right]}{\mu_{\kappa}},
$$

with utility normalisation $u^{\prime}(w)=1$.

Proposition 4. For any risk characteristics $(L, \tilde{\kappa})$, there exists $\bar{\lambda}>0$, such that the asymptotic insurance coverage $I^{*}$ is positive if $\lambda<\bar{\lambda}$.

Proposition 4 shows that a correlated catastrophic risk generates a positive level of coverage in equilibrium, if transaction costs are not too large. In other words, there is no intrinsic barriers to insurance coverage for such risks.

We may consider a more tractable expression for a risk $\tilde{\kappa}$ that is small compared with aggregate wealth $w$, with a nevertheless systemic nature due to $\rho>0$. Approximating $u^{\prime}(w-\tilde{\kappa} L)$ at the first order around $u^{\prime}(w)$. This gives

$$
\psi(0)=(1+\lambda)[1+A(w) L \rho] .
$$

This approximation ${ }^{16}$ shows that an increase in the correlation coefficient $\rho$ reduces the equilibrium coverage $I^{*}$ since it makes insurance more expensive. An increase in $L$ has a potentially ambiguous effect on $I^{*}$. On the one hand, the representative risk-averse individual provides the capital that sustain the insurance scheme, and thus higher losses increase the required premium, which tends to reduce the equilibrium coverage. On the other hand, an increase on the risk exposure of policyholders tends to increase their insurance demand. For static comparative purposes, we may write the asymptotic equilibrium indemnity $I^{*}(L, \rho, \lambda)$ as a function of the loss level, the correlation coefficient and the loading factor, and similarly $\beta^{*}(L, \rho, \lambda)=I^{*}(L, \rho, \lambda) / L$.

Proposition 5. Assume that $u$ is DARA and $\lambda>0$. Then, under the approximated pricing rule (19), there exist a threshold $\underline{L}(\lambda, \rho)>0$, with

\footnotetext{
${ }^{16}$ Equation 19 is an approximation of $\psi(0)$ by default when $u^{\prime \prime \prime}>0$ that would lead to over-estimate the coverage rates. Numerical simulations, not reported here for the sake of brevity but available from the authors upon request, show that approximating the true price with $(19)$ produces reasonable levels of errors (a few percentage points) for the calibrations considered in the next section.
} 
$\partial \underline{L} / \partial \lambda<0$ and $\partial \underline{L} / \partial \rho<0$ such that $I^{*}(L, \rho, \lambda)>0$ if $L>\underline{L}(\lambda, \rho)$. When $L>\underline{L}(\lambda, \rho)$, we have

$$
\frac{\partial \beta^{*}}{\partial L}>0, \frac{\partial \beta^{*}}{\partial \rho}<0, \frac{\partial \beta^{*}}{\partial \lambda}<0 .
$$

In the presence of transaction costs $(\lambda>0)$, the coverage rate is positive only when the loss is sufficiently high and for $\lambda$ given, an increase in the individual loss $L$ (respect. coefficient of correlation $\rho$ ) stimulates (reduces) insurance demand and leads to an increase (a decrease) in the coverage rate $\beta^{*}(L, \rho, \lambda)$. Importantly, formula $(19)$ is valid for risks that have a systemic nature $(\rho>0)$ but that remain small compared to aggregate wealth ${ }^{17}$ This may be unduly restrictive, and thus we revert to the more general expression (18) to perform the simulations of the next section.

In contrast with Propositions 4 and 5 , that provide an optimistic view on the ability of insurance markets to cover catastrophic risks, transaction costs, market failures, and behavioural biases, deliberately ignored here, are likely to play a significant role when the observed coverage of low-probability high-severity risks is weak. In particular, limited liability and concerns about default may play an important role as suggested in Zanjani (2002) and Cummins et al. (2002). High transaction costs between insurers and reinsurers also limit the ability of insurers to pay for "the big one" as suggested in Froot (2001) and Niehaus (2002). Imperfect information on risk exposure or biases in beliefs about such risks may also deter individuals from purchasing insurance. In other words, the frequently observed under-coverage of catastrophic risks through private insurance mechanisms is likely to result from such market imperfections rather than from an hypothetical intrinsic weakness of insurance mechanism to deal with such risks. From an economic policy standpoint, this suggests that reducing such market imperfections should be right at the top of the agenda. Of course, such a conclusion makes sense only when the insurance industry is sufficiently strong to manage the financial transfer of catastrophic risks.

\section{Numerical application}

This section conducts numerical simulations that illustrate our theoretical results. We assume that policyholders display harmonic absolute risk aver-

\footnotetext{
${ }^{17}$ This restriction on the size of the catastrophe need not be as restrictive as it seems. For example, the Japanese government expects the total cost of the Fukushima-Daiichi accident in 2011 to amount to a 177 billion euros bill. An important part of this cost is systemic since it affects many people at once, but its size, when compared to a 4300 billion euros annual GDP, remains limited.
} 
sion (HARA) preferences, characterised by (2) and a coefficient of relative risk aversion

$$
R(x)=x\left(\eta+\frac{x}{\gamma}\right)^{-1} .
$$

Using the CRRA specification, studies on individual data, such as Levy (1994) and Szpiro (1986), have isolated a plausible range between 1 and 5 for the index of relative risk aversion. We therefore perform simulations over this plausible range of values. In particular, we calibrate the coefficient of relative risk aversion in the no-loss state $R(w) \equiv \bar{R}=3$ and we let the coefficient of relative aversion in the loss state $R(w-L) \equiv \underline{R}$, vary between 1 and 5 . When $\bar{R}=\underline{R}$, the HARA specification boils down the CRRA utility function.

Solving the agent's optimisation program in the HARA case and with the pricing rule (14) yields a closed form solution for all acceptable values of $p$

$$
I^{* *}(p)=\frac{L+(\eta \gamma+w)(\chi(p)-1)}{1+\psi(p) p(\chi(p)-1)}, \quad \text { where } \quad \chi(p)=\left[\frac{1-\psi(p) p}{\psi(p)(1-p)}\right]^{\frac{1}{\gamma}},
$$

which indeed converges to the value given by (17) when $p \rightarrow 0$. Importantly, the results of the numerical simulations do not rely on any any approximation. We nevertheless report the error that one would be making by approximating the exact optimum $I^{* *}(p)$ by its limit counterpart $I^{*}$. It turns out that this error is fairly small in all scenarios considered.

In order to simulate these optimal coverage levels, we calibrate a beta distribution for the variable $\tilde{\kappa}$. We consider a catastrophe of probability $\pi=$ $1 \%$ and $\mu_{\kappa}=0.1$. That is, the fraction of the population expected to incur a loss in case of catastrophe is $10 \%$. The individual loss probability is therefore $p=0.1 \%$. In addition, the variance of $\tilde{\kappa}$ is set at $\sigma_{\tilde{\kappa}}^{2}=0.001$, which gives a coefficient of correlation $\rho=0.109$. We also compute the premium $\psi^{* *}(p)$ associated with the optimal levels of coverage and the relative difference

$$
\varepsilon(p)=\frac{I^{*}-I^{* *}(p)}{I^{* *}(p)}
$$

between the true optimal coverage $I^{* *}(p)$ and its asymptotic value $I^{*}$, characterised by Equation (21) and Proposition 3, respectively.

These simulations are reported in Table 1. The size of the loss $L$ varies across lines from 200,000 euros to 800,000 euros to capture the monetary consequences of a severe accident. Initial wealth $w$ is set at one million euros, which represents roughly the average lifetime discounted earnings of a French citizen. We assume $\bar{R}=3$ and $\underline{R}$ varies across columns between 1 and 5 . Finally, the insurance provider's costs are captured by a proportional loading factor $\lambda=0.3$. 


\begin{tabular}{|c|c|c|c|c|c|c|}
\hline \multicolumn{1}{|c|}{$\underline{R}$} & & 1 & 2 & 3 & 4 & 5 \\
\hline \multirow{3}{*}{200,000} & $I^{* *}$ & 39,016 & 84,616 & 96,098 & 101,285 & 104,235 \\
& $\psi^{* *}$ & 1.38 & 1.39 & 1.39 & 1.39 & 1.39 \\
& $\varepsilon$ & $(0.89)$ & $(0.19)$ & $(0.11)$ & $(0.08)$ & $(0.06)$ \\
\hline \multirow{3}{*}{400,000} & $I^{* *}$ & 252,130 & 270,411 & 275,869 & 278,490 & 280,028 \\
& $\psi^{* *}$ & 1.47 & 1.47 & 1.47 & 1.47 & 1.47 \\
& $\varepsilon$ & $(0.12)$ & $(0.05)$ & $(0.03)$ & $(0.02)$ & $(0.02)$ \\
\hline \multirow{3}{*}{600,000} & $I^{* *}$ & 443,172 & 452,505 & 455,460 & 456,909 & 457,770 \\
& $\psi^{* *}$ & 1.56 & 1.56 & 1.56 & 1.56 & 1.56 \\
& $\varepsilon$ & $(0.05)$ & $(0.02)$ & $(0.01)$ & $(0.01)$ & $(0.01)$ \\
\hline \multirow{3}{*}{800,000} & $I^{* *}$ & 629,572 & 633,554 & 634,854 & 635,499 & 635,885 \\
& $\psi^{* *}$ & 1.64 & 1.64 & 1.64 & 1.64 & 1.64 \\
& $\varepsilon$ & $(0.02)$ & $(0.01)$ & $(0.00)$ & $(0.00)$ & $(0.00)$ \\
\hline
\end{tabular}

Table 1: Optimal coverage $I^{* *}$ in euros, optimal loading $\psi^{* *}$, and relative error $\varepsilon$, at $\pi=1 \%, \mu_{\kappa}=0.1$ and $\sigma_{\kappa}^{2}=0.001$ (hence $p=0.001$ and $\rho=0.109$ ).

Optimal coverage increases with the size of the loss and with the coefficient of risk aversion $\underline{R}$. For a given level of risk aversion $\underline{R}=3$, it rises from 96, 098 to 634,854 euros when $L$ increases from 200, 000 to 800, 000 euros. Fixing the loss at 800,000 euros results in a modest increase in optimal coverage from 629,572 euros when $\underline{R}=1$ to 635,885 euros when $\underline{R}=5$. The comparative statics with respect to $\underline{R}$ is of particular interest because it summarises two distinct effects. One the one hand, higher risk aversion pushes toward higher demand levels. On the other hand, it also also yields higher supply prices through the risk premium required in equilibrium. In our simulation, the demand effect dominates the price effect because the risk considered are very large at the individual level but remain limited compared to the overall wealth of the economy.

Coverage rates are limited for lower losses levels, but they increase rapidly with the size of the loss. Because we have fixed relative risk aversion in the loss state $\underline{R}=(w-L) A(w-L)$, the index of absolute risk aversion becomes large as the loss $L$ gets closer to initial wealth $w$. These higher levels of absolute risk aversion in the loss state explain the higher coverage rates observed for higher loss levels, as we have shown in the previous sections.

The second number of each box represents the total loading $\psi^{* *}$ associated with the optimal coverage. For $L=800,000$ and $\underline{R}=3$, policyholders pay a premium equal to 1.64 times their expected loss. Since the exogenous loading factor $\lambda$ was set at 0.3 , this implies that the cost of capital is responsible 
for a 34 percentage points increase in the loading factor. The loading factor increases with both $L$ and $\underline{R}$ but the effect of $\underline{R}$ is quantitatively very limited because $\underline{R}$ mainly affects the insurance cost through the coefficient of absolute risk aversion. Since $A(w)=R(w) / w$, a unit change in $R(w)$ only leads to limited changes in $A(w)$. In contrast, an increase in $L$ directly affect the cost of insurance as Equation (15) shows.

Finally, bracketed numbers report the error in percent, $\varepsilon(p)$, that one would be making by approximating $I^{* *}(p)$ with $I^{*}$. It varies between $0.89 \%$ when $L=200,000$ euros and $\underline{\mathrm{R}}=1$ and negligible values in most scenarios. These low errors confirm that $I^{*}$ is an interesting quantity to look at when considering insurance decisions for low-probability risks. ${ }^{1919}$

\begin{tabular}{|c|c|c|c|c|c|c|}
\hline$\underline{R}$ & & 1 & 2 & 3 & 4 & 5 \\
\hline \multirow{3}{*}{200,000} & $I^{* *}$ & 24,920 & 75,633 & 88,318 & 94,031 & 97,274 \\
& $\psi^{* *}$ & 1.42 & 1.42 & 1.42 & 1.42 & 1.42 \\
& $\varepsilon$ & $(1.66)$ & $(0.22)$ & $(0.12)$ & $(0.09)$ & $(0.07)$ \\
\hline \multirow{3}{*}{400,000} & $I^{* *}$ & 232,569 & 253,217 & 259,308 & 262,212 & 263,910 \\
& $\psi^{* *}$ & 1.53 & 1.53 & 1.53 & 1.53 & 1.53 \\
& $\varepsilon$ & $(0.15)$ & $(0.06)$ & $(0.04)$ & $(0.03)$ & $(0.02)$ \\
\hline \multirow{3}{*}{600,000} & $I^{* *}$ & 415,619 & 425,760 & 428,879 & 430,385 & 431,270 \\
& $\psi^{* *}$ & 1.65 & 1.65 & 1.65 & 1.65 & 1.65 \\
& $\varepsilon$ & $(0.07)$ & $(0.03)$ & $(0.02)$ & $(0.01)$ & $(0.01)$ \\
\hline \multirow{3}{*}{800,000} & $I^{* *}$ & 591,744 & 595,498 & 596,660 & 597,222 & 597,552 \\
& $\psi^{* *}$ & 1.77 & 1.77 & 1.77 & 1.77 & 1.77 \\
& $\varepsilon$ & $(0.03)$ & $(0.01)$ & $(0.00)$ & $(0.00)$ & $(-0.00)$ \\
\hline
\end{tabular}

Table 2: Optimal coverage $I^{* *}$ in euros, optimal loading $\psi^{* *}$, and relative error $\varepsilon$, at $\pi=1 \%, \mu_{\kappa}=0.1$ and $\sigma_{\kappa}^{2}=0.005$ (hence $p=0.001$ and $\rho=0.149$ ).

As a final exercise, Table 2 considers the case of a higher variance $\sigma_{\kappa}^{2}=$ 0.005 and therefore of a higher correlation $\rho=0.149$. The role of the systemic component of the risk is well illustrated here. With a higher variance $\sigma_{\kappa}^{2}$, it

\footnotetext{
${ }^{18}$ Considering higher levels of loss probability would indeed give rise to higher errors. Considering $\pi=0.1$ instead of $\pi=0.01$ for, would results in approximation errors lower than $8.55 \%$ and lower than $2 \%$ in 17 out of the 20 scenarios of Table 1 .

${ }^{19}$ In addition, large catastrophes often affect people with sometimes widely different (but small) probabilities. Our result suggests that such differences in risk exposure may actually result in very limited differences in optimal coverage values. Similarly, conflicting expert opinions concerning the true probability of a catastrophe would also be irrelevant for the choice of an optimal level of coverage, as long as experts agree that the probability $\pi$ is very small.
} 
is more costly to provide insurance, so all loadings are higher. At the same time, the expected probability of loss for a given individual remains constant, hence the lower levels of coverage optimally purchased.

\section{Conclusion}

The purpose of this paper was to analyse the key determinants of the optimal insurance for catastrophic risks associated with low-probability high-severity events. Considering the limit case of a vanishingly low probability of loss, we have analysed how insurance demand is affected by the degree of risk aversion when individuals face large-scale risks, and we have characterised the asymptotic insurance coverage. On the supply side, the correlation of losses that characterises catastrophic risks results in a risk premium in equilibrium. Added to transaction costs, this translates into higher prices for policyholders.

This cost is a well-known impediment to the insurability of catastrophic risks. Our analysis however, demonstrates that this impediment alone hardly explains the uninsurability of low-probability high-severity risks. In particular, under reasonably low transaction costs and complete financial markets, the cost of capital itself should not prevent the insurability of catastrophic risks. The larger the loss incurred by victims and/or the lower the correlation between individual losses, the larger the equilibrium rate of insurance coverage. Insurance transaction costs and capital market imperfections, increasing further the cost and lowering the availability of capital therefore play an important role in explaining the failure to insure catastrophic risks. Other market failures, such as asymmetries of information, behavioural biases or lack of competition may also contribute to the absence of insurance markets for the more systemic lines of risk.

Governments have an important role to play in the reduction of these transaction costs in interaction with capital markets. This may go through policy measures that reduce transaction costs or capital costs for risks that would otherwise be uninsurable. Sometimes criticised for its cost in the federal budget, the U.S. National Flood Insurance Program nevertheless aims at lowering insurance transaction costs, by making underwriting and claims handling easier, while also encouraging local communities to take prevention measures that reduce future flood damage. In the field of nuclear risk, international conventions have endorsed a common nuclear corporate liability law, thereby reducing the claims handling costs in the case of a nuclear accident. As for the reduction of capital costs, insurance pools provide examples of what proactive policies can do. This is the case for natural disaster risk 
(e.g., Flood Re in the UK, or the Caribbean Catastrophe Risk Insurance Facility for hurricanes and earthquakes, to mention just two examples), for large-scale terrorism risk (e.g., the GAREAT pool in France), and for the nuclear risk in almost all countries with nuclear power plants. By contrast, failing to pass onto policyholders the cost of capital due to the systemic component of catastrophic risks would send biased signals about the social cost of risk. The consequences of such an approach on risk prevention behaviours and on the perception of the size of cross-subsidisation between more or less exposed individuals are sometimes at the origin of criticisms made towards state-sponsored insurance regimes, such as the natural disaster insurance regimes in France and Spain. 


\section{Proofs}

\subsection{Proof of Proposition 1}

From equation (2), we have

$$
C_{p}^{\prime}\left(0_{+}, \tilde{\ell}\right)=\frac{u(w)-u(w-\mathbb{E} \tilde{\ell})}{u^{\prime}(w)}=\mathbb{E}\left[\int_{w-\tilde{\ell}}^{w} \frac{u^{\prime}(x)}{u^{\prime}(w)} d x\right] .
$$

Since

$$
u^{\prime}(x)=u^{\prime}(w)-\int_{x}^{w} u^{\prime \prime}(t) d t
$$

for all $x \in[w-\bar{L}, w]$, we may write

$$
\begin{aligned}
C_{p}^{\prime}\left(0_{+}, \tilde{\ell}\right) & =\mathbb{E} \tilde{\ell}-\mathbb{E}\left[\int_{w-\tilde{\ell}}^{w}\left[\int_{x}^{w} \frac{u^{\prime \prime}(t)}{u^{\prime}(w)} d t\right] d x\right] \\
& =\mathbb{E} \tilde{\ell}+\mathbb{E}\left[\int_{w-\tilde{\ell}}^{w}\left[\int_{x}^{w} A(t) \frac{u^{\prime}(t)}{u^{\prime}(w)} d t\right] d x\right],
\end{aligned}
$$

and thus,

$$
\begin{aligned}
\theta(0+, \tilde{\ell}) & =\frac{C_{p}^{\prime}\left(0_{+}, \tilde{\ell}\right)-\mathbb{E} \tilde{\ell}}{(\mathbb{E} \tilde{\ell})^{2}} \\
& =\frac{1}{(\mathbb{E} \tilde{\ell})^{2}} \mathbb{E}\left[\int_{w-\tilde{\ell}}^{w}\left[\int_{x}^{w} A(t) \frac{u^{\prime}(t)}{u^{\prime}(w)} d t\right] d x\right]
\end{aligned}
$$

Integrating by parts three times and making the change of variable $x=w-\ell$ gives

$$
\begin{aligned}
\theta\left(0_{+}, \tilde{\ell}\right) & =\frac{1}{(\mathbb{E} \tilde{\ell})^{2}} \int_{0}^{\bar{L}}\left[\int_{w-\ell}^{w}(x-w+\ell) A(x) \frac{u^{\prime}(x)}{u^{\prime}(w)} d x\right] f(\ell) d \ell \\
& =\frac{1}{(\mathbb{E} \tilde{\ell})^{2}}\left\{\int_{w-\bar{L}}^{w}(x-w+\ell) A(x) \frac{u^{\prime}(x)}{u^{\prime}(w)} d x-\int_{0}^{\bar{L}} F(\ell)\left[\int_{w-\ell}^{w} A(x) \frac{u^{\prime}(x)}{u^{\prime}(w)} d x\right] d \ell\right\} \\
& =\frac{1}{(\mathbb{E} \tilde{\ell})^{2}}\left\{\int_{w-\bar{L}}^{w}\left[x-w+\bar{L}-\int_{w-x}^{\bar{L}} F(\ell) d \ell\right] A(x) \frac{u^{\prime}(x)}{u^{\prime}(w)} d x\right\} .
\end{aligned}
$$

Using

$$
u^{\prime}(x)=u^{\prime}(w) \exp \left\{\int_{x}^{w} A(x) d x\right\},
$$

completes the first part of the proof. Integrating by parts twice then gives

$$
\int_{w-\bar{L}}^{w}\left[x-w+\bar{L}-\int_{w-x}^{\bar{L}} F(\ell) d \ell\right] d x=\frac{1}{2}(\mathbb{E} \tilde{\ell})^{2},
$$

hence

$$
\int_{w-\bar{L}}^{w} k(x) d x=1
$$




\subsection{Proof of Corollary 1}

The corollary follows straightforwardly from

$$
\exp \left\{\int_{x}^{w} A(t) d t\right\} \geq 1
$$

\subsection{Proof of Proposition 2}

Let $\nu(x)=x-w+\bar{L}-\int_{w-x}^{\bar{L}} F(\ell) d \ell>0$ for all $x \in[w-\bar{L}, w]$, with $\nu(w)=$ $\bar{L}-\int_{0}^{\bar{L}} F(\ell) d \ell=\mathbb{E} \tilde{\ell}$ and $f^{\prime}(\bar{L})<0$. Equation 23 is therefore written as

$$
\theta\left(0_{+}, \tilde{\ell}\right)=\frac{1}{(\mathbb{E} \tilde{\ell})^{2}} \int_{w-\bar{L}}^{w} \eta(x) A(x) \frac{u^{\prime}(x)}{u^{\prime}(w)} d x .
$$

Hence

$$
\begin{aligned}
\frac{d\left[\nu(x) u^{\prime}(x)\right]}{d x} & =u^{\prime}(x) \nu^{\prime}(x)+\nu(x) u^{\prime \prime}(x) \\
& =u^{\prime}(x)\left[\nu^{\prime}(x)-\frac{\nu(x)}{x} R(x)\right] \quad x \in[w-\bar{L}, w] .
\end{aligned}
$$

$d\left[\nu(x) u^{\prime}(x)\right] / d x>0$ if $\varepsilon(x) \equiv \frac{\nu^{\prime}(x) x}{\nu(x)}>R(x)$, and

$$
\varepsilon(x)=\frac{x[1-F(w-x)]}{\int_{w-x}^{\bar{L}}[1-F(\ell)] d \ell} .
$$

Since $\mathbb{E} \tilde{\ell}=\bar{L}-\int_{0}^{\bar{L}} F(\ell) d \ell$, we have $\varepsilon(w)=w / \mathbb{E} \tilde{\ell}$. Assuming $f(\ell) \rightarrow 0$ when $\ell \rightarrow \bar{L}$ and $f^{\prime}(\bar{L})<0$ and applying L'Hôpital's rule twice, we obtain $\varepsilon(x) \rightarrow+\infty$ when $x \rightarrow w-\bar{L}$. Assuming that $\varepsilon(x)$ is monotonic, and therefore decreasing, implies

$$
\begin{aligned}
\varepsilon(x) & >\varepsilon(w) \quad \text { if } \quad x<w . \\
& =\frac{w}{\mathbb{E} \tilde{\ell}}
\end{aligned}
$$

A sufficient condition for $d\left[\nu(x) u^{\prime}(x)\right] / d x>0$ is therefore

$$
\frac{w}{\mathbb{E} \tilde{\ell}}>\bar{\gamma}
$$

where $\bar{\gamma}=\max \{R(x), x \in[w-\bar{L}, w]\}$. In this case, we have

$$
\begin{aligned}
\nu(x) u^{\prime}(x) & <\nu(w) u^{\prime}(w) \quad \text { if } \quad x \in[w-\bar{L}, w] \\
& =\mathbb{E} \tilde{\ell} u^{\prime}(w),
\end{aligned}
$$


and consequently

$$
\theta\left(0_{+}, \tilde{\ell}\right)<\frac{1}{\mathbb{E} \tilde{\ell}} \int_{w-\bar{L}}^{w} A(x) d x .
$$

Let $\bar{A}=1 / L \int_{w-\bar{L}}^{w} A(x) d x$. We then obtain

$$
\theta\left(0_{+}, \tilde{\ell}\right)<\frac{\bar{L}}{\mathbb{E} \tilde{\ell}} \bar{A} .
$$

Using $C_{p}^{\prime \prime}<0$ and $C\left(0_{+}, \tilde{\ell}\right)=0$ with equation (1) allows us to write

$$
\begin{aligned}
C(p, \tilde{\ell}) & <C^{\prime}\left(0_{+}, \tilde{\ell}\right) p \\
& =p \mathbb{E} \tilde{\ell}\left[1+\theta\left(0_{+}, \tilde{\ell}\right) \mathbb{E} \tilde{\ell}\right] \\
& <p \mathbb{E} \tilde{\ell}[1+\bar{L} \bar{A}] .
\end{aligned}
$$

\subsection{Proof of Corollary 3}

A simple calculation shows that Proposition 1 and Corollary 1 can be rewritten as

Corollary 4. When $\tilde{\ell}=L$ with probability 1 , we have

$$
\theta\left(0_{+}, \tilde{\ell}\right)=\frac{1}{2} \int_{w-L}^{w}\left[k(x) A(x) \exp \left\{\int_{x}^{w} A(t) d t\right\}\right] d x>\frac{1}{2} \int_{w-L}^{w} k(x) A(x) d x
$$

where

$$
k(x)=\frac{2[x-(w-L)]}{L^{2}}>0
$$

and

$$
\int_{w-L}^{w} k(x) d x=1
$$

Using Lemma (1) then shows that

$$
d\left(0_{+}, L\right) \leq \frac{L}{2} \int_{w-L}^{w} \frac{k(x)}{x} R(x) d x
$$

is a sufficient condition insurance take-up to be positive. If $R(x)$ is non decreasing, then

$$
\begin{aligned}
\frac{L}{2} \int_{w-L}^{w} \frac{k(x)}{x} R(x) d x & \geq \frac{L R(w-L)}{2} \int_{w-L}^{w} \frac{k(x)}{x} d x \\
& =\frac{R(w-L)}{L} \int_{w-L}^{w} \frac{x-(w-L)}{x} d x \\
& =R(w-L)\left[1-\left(\frac{w-L}{L}\right) \ln \frac{w}{w-L}\right] \\
& \equiv \Psi(L) \quad L \in[0, w] .
\end{aligned}
$$

Noticing that $\lim _{L \rightarrow w} \psi(L)=\lim _{x \rightarrow 0} R(x)$ provides the result. 


\subsection{Coefficient of correlation $\rho$}

Let $\tilde{L}_{i}$ and $\tilde{L}_{j}$ be two random variables that represent the losses of individuals $i$ and $j$. Conditionally on a realization $\kappa$ of the random variable $\tilde{\kappa}$, losses are assumed identically and independently distributed, hence

$$
\tilde{L}_{i} \tilde{L}_{j} \mid \kappa=\left\{\begin{array}{cll}
L^{2} & \text { with probability } & \pi \kappa^{2} \\
0 & \text { with probability } & 1-\pi \kappa^{2}
\end{array} .\right.
$$

As a consequence $\mathbb{E}\left(\tilde{L}_{i} \tilde{L}_{j} \mid \kappa\right)=L^{2} \pi \kappa^{2}$ and $\mathbb{E}\left(\tilde{L}_{i} \tilde{L}_{j}\right)=L^{2} \pi \mathbb{E} \tilde{\kappa}^{2}$. Similarly,

$$
\tilde{L}_{i} \mid \kappa=\left\{\begin{array}{lll}
L & \text { with probability } & \pi \kappa \\
0 & \text { with probability } & 1-\pi \kappa
\end{array}\right.
$$

for all $i$, hence $\mathbb{E} \tilde{L}_{i}=L \pi \mu_{\kappa}$ and $\mathbb{E} \tilde{L}_{i} \mathbb{E} \tilde{L}_{j}=\left(L \pi \mu_{\kappa}\right)^{2}$. The co-variance between two losses is therefore written as

$$
\operatorname{cov}\left(\tilde{L}_{i}, \tilde{L}_{j}\right)=L^{2} \pi\left[\mathbb{E} \tilde{\kappa}^{2}-\pi \mu_{\kappa}^{2}\right]
$$

Also, since

$$
\tilde{L}_{i}^{2} \mid \kappa=\left\{\begin{array}{cll}
L^{2} & \text { with probability } & \pi \kappa \\
0 & \text { with probability } & 1-\pi \kappa
\end{array},\right.
$$

implies $\mathbb{E}\left(\tilde{L}_{i}{ }^{2}\right)=\pi L^{2} \mu_{\kappa}$, we find the variance of $\tilde{L}_{i}$

$$
\operatorname{Var}\left(\tilde{L}_{i}\right)=L^{2} \pi \mu_{\kappa}\left(1-\pi \mu_{\kappa}\right)
$$

Since $\operatorname{Var}\left(\tilde{L}_{i}\right)=\operatorname{Var}\left(\tilde{L}_{j}\right)$ for all $i$, the coefficient of correlation is finally equal to

$$
\begin{aligned}
\rho & =\frac{\operatorname{cov}\left(\tilde{L}_{i}, \tilde{L}_{j}\right)}{\operatorname{Var}\left(\tilde{L}_{i}\right)} \\
& =\frac{\mathbb{E} \tilde{\kappa}^{2}-\pi \mu_{\kappa}^{2}}{\mu_{\kappa}\left(1-\pi \mu_{\kappa}\right)} \\
& =\frac{\sigma_{\kappa}^{2}+\mu_{\kappa}^{2}(1-\pi)}{\mu_{\kappa}\left(1-\pi \mu_{\kappa}\right)},
\end{aligned}
$$

where the last line is obtained using $\sigma_{\kappa}^{2}=\mathbb{E} \tilde{\kappa}^{2}-\mu_{\kappa}^{2}$. 


\subsection{Proof of Proposition 4}

When $\lambda=0$, inequality (16) is rewritten as

$$
\frac{\mathbb{E}\left[\tilde{\kappa} u^{\prime}(w-\tilde{\kappa} L)\right]}{\mu_{\kappa}} \leq u^{\prime}(w-L) .
$$

Since $u^{\prime \prime}<0$, we have

$$
u^{\prime}(w-\kappa L)<u^{\prime}(w-L) \text { for all } \kappa \in[0,1],
$$

and hence

$$
\mathbb{E}\left[\tilde{\kappa} u^{\prime}(w-\tilde{\kappa} L)\right]<\mu_{\kappa} u^{\prime}(w-L),
$$

which gives (24). (16) also holds when $\lambda$ is not too large.

\subsection{Proof of Proposition 5}

Using Proposition 3 with equations $(16)$ and $(19)$ gives $I^{*}>0$ if

$$
\varphi(L) \equiv u^{\prime}(w-L)-x(L, \lambda, \rho)>0,
$$

where $x(L, \lambda, \rho) \equiv(1+\lambda)(1+A(w) L \rho)$. Using $u^{\prime}(w)=1$, we obtain $\varphi(0)=$ $u^{\prime}(w)-x(0, \lambda, \rho)=-\lambda<0$, and

$$
\begin{array}{r}
\varphi^{\prime}(L)=-u^{\prime \prime}(w-L)-(1+\lambda) A(w) \rho \\
\varphi^{\prime \prime}(L)=u^{\prime \prime \prime}(w-L)>0
\end{array}
$$

The function $\varphi(L)$ is therefore convex with $\varphi(0)<0$ and $\varphi(\bar{L})>0$ if $u^{\prime}(w-$ $\bar{L})>(1+\lambda)[1+A(w) \bar{L} \rho] . \varphi(L)=0$ hence defines $\underline{L}(\lambda, \rho)$ with $\bar{L}(\lambda, \rho)=0$ if $\lambda=0$ and $\bar{L}(\lambda, \rho)>0$ if $\lambda>0$. When $L>\underline{L}(\lambda, \rho)$, (i.e. $I^{*}>0$ ), Equation (17) gives

$$
\beta^{*}=1+\frac{u^{\prime-1}(x(L))-w}{L},
$$

for given values of $\lambda$ and $\rho$. Let $z(L)=u^{-1}(x(L))$. Since DARA implies $u^{\prime \prime \prime}>0, u^{\prime-1}$ is decreasing and convex and since $x(L)$ is linear, $z(L)$ is also decreasing and convex. From (25), we obtain

$$
\begin{aligned}
\frac{\partial \beta^{*}}{\partial L} & =\left[\frac{L x^{\prime}(L)}{u^{\prime \prime}\left(u^{\prime-1}(x(L))\right)}-u^{\prime-1}(x(L))+w\right] / L^{2} \\
& =\frac{L z^{\prime}(L)-z(L)+w}{L^{2}} .
\end{aligned}
$$

Using $w>=z(0)$ and the convexity of function $z(L)$ yields

$$
L z^{\prime}(L)-z(L)+w>=L z^{\prime}(L)-z(L)+z(0)>0,
$$

which gives $\partial \beta^{*} / \partial L>0$ when $L \geq \bar{L}(\lambda, \rho)$. The comparative statics $\partial \beta^{*} / \partial \rho<$ 0 and $\partial \beta^{*} / \partial \lambda<0$ are obtained by noticing that an increase in either $\lambda$ or $\rho$ translates into an increase in $x(L) . u^{-1}$ decreasing then provides the results. 


\section{Bibliography}

\section{References}

Arrow, K. J. (1963). Liquidity preference, lecture vi in" lecture notes for economics 285, the economics of uncertainty". undated, Stanford University, (pp. 33-53).

Barro, R. J. (2006). Rare disasters and asset markets in the twentieth century. The Quarterly Journal of Economics, 121(3), 823-866.

Charpentier, A. \& Le Maux, B. (2014). Natural catastrophe insurance: How should the government intervene? Journal of Public Economics, 115, 1-17.

Cummins, J. D., Doherty, N., \& Lo, A. (2002). Can insurers pay for the "big one"? measuring the capacity of the insurance market to respond to catastrophic losses. Journal of Banking \& Finance, 26, 557-583.

Eeckhoudt, L., Schieber, C., \& Schneider, T. (2000). Risk aversion and the external cost of a nuclear accident. Journal of environmental Management, 58(2), 109-117.

Farhi, E. \& Gabaix, X. (2015). Rare disasters and exchange rates. The Quarterly Journal of Economics, 131(1), 1-52.

Froot, K. A. (2001). The market for catastrophe risk: a clinical examination. Journal of Financial Economics, 60(2), 529-571.

Gollier, C. (1995). The comparative statics of changes in risk revisited. Journal of Economic Theory, 66(2), 522-535.

Gollier, C. (2004). The economics of risk and time. MIT press.

Gourio, F. (2012). Disaster risk and business cycles. American Economic Review, 102(6), 2734-66.

Grislain-Letrémy, C. (2018). Natural disasters: exposure and underinsurance. Annals of Economics and Statistics/Annales d'Économie et de Statistique, (129), 53-83.

Hertwig, R., Barron, G., Weber, E. U., \& Erev, I. (2004). Decisions from experience and the effect of rare events in risky choice. Psychological science, $15(8), 534-539$. 
Ikefuji, M., Laeven, R. J., Magnus, J. R., \& Muris, C. (2015). Expected utility and catastrophic consumption risk. Insurance: Mathematics and Economics, 64, 306-312.

Jaffee, D. M. \& Russell, T. (1997). Catastrophe insurance, capital markets, and uninsurable risks. Journal of Risk and Insurance, (pp. 205-230).

Kousky, C. \& Cooke, R. (2012). Explaining the failure to insure catastrophic risks. The Geneva Papers on Risk and Insurance-Issues and Practice, 37(2), 206-227.

Kousky, C., Michel-Kerjan, E. O., \& Raschky, P. (2013). Does federal disaster assistance crowd out private demand for insurance. Risk Management and Decision Processes Center The Wharton School, University of Pennsylvania, 10 .

Kunreuther, H. (1973). Recovery From Natural Disasters: Insurance or Federal Aid? Washington.

Kunreuther, H., Novemsky, N., \& Kahneman, D. (2001). Making low probabilities useful. Journal of Risk and Uncertainty, 23(2), 103-120.

Kunreuther, H. \& Slovic, P. (1978). Economics, psychology, and protective behavior. The American Economic Review, 68(2), 64-69.

Mossin, J. (1968). Aspects of rational insurance purchasing. The Journal of Political Economy, (pp. 553-568).

Niehaus, G. (2002). The allocation of catastrophe risk. Journal of Banking Ef Finance, 26(2-3), 585-596.

Pratt, J. W. (1964). Risk aversion in the small and in the large. Econometrica: Journal of the Econometric Society, (pp. 122-136).

Raschky, P. A., Schwarze, R., Schwindt, M., \& Zahn, F. (2013). Uncertainty of governmental relief and the crowding out of flood insurance. Environmental and Resource Economics, 54(2), 179-200.

Raviv, A. (1979). The design of an optimal insurance policy. The American Economic Review, 69(1), 84-96.

Rietz, T. A. (1988). The equity risk premium a solution. Journal of monetary Economics, 22(1), 117-131. 
Rothschild, M. \& Stiglitz, J. E. (1971). Increasing risk ii: Its economic consequences. Journal of Economic theory, 3(1), 66-84.

Schlesinger, H. (2013). The theory of insurance demand. In Handbook of insurance (pp. 167-184). Springer.

Weitzman, M. L. (2009). On modeling and interpreting the economics of catastrophic climate change. The Review of Economics and Statistics, 91(1), 1-19.

Zanjani, G. (2002). Pricing and capital allocation in catastrophe insurance. Journal of Financial Economics, 65(2), 283-305. 